\title{
Study of radiation properties in Taylor distribution uniform spaced backfire antenna arrays
}

\author{
Amer Tawfeeq Abed \\ Lecturer in Communication Eng. Department of Antennas and RF circuits Design, AL-MAMON University College, $14^{\text {th }}$ Ramadan \\ Street Baghdad- Iraq
}

Email address:

engamer1963@yahoo.com

\section{To cite this article:}

Amer Tawfeeq Abed. Study of Radiation Properties in Taylor Distribution Uniform Spaced Backfire Antenna Arrays. American Journal of Electromagnetics and Applications. Vol. 2, No. 3, 2014, pp. 23-26. doi: 10.11648/j.ajea.20140203.11

\begin{abstract}
This paper is the last part in studying the directivity for Linear Long Backfire Antenna arrays, the research will focus on another type of non linear excitation (Taylor )excitation uniform space long backfire antenna array especially the directivity and SLL max (side lobe level maximum )properties of the radiation patterns and plot the relation between the length of the arrays and the directivity. The effect of excitation coefficients values for all elements in the arrays on radiation properties will be studied in all types of non uniform excitation arrays. At last the directivity and SLL for three types of non uniform distribution (Taylor, Chebyshev and Binomial) will be compered .
\end{abstract}

Keyword: Taylor array, Radiation properties, Backfire Antenna

\section{Introduction}

Taylor one parameter method is more applicable to the line source distribution. To apply this method to discrete array antenna pattern synthesis, the aperture distribution must be sampled [1]. The array factor for Taylor array is the same as the array factor for binomial and edge arrays in eq. (1). The amplitude current excitation coefficient can be calculated from the source distribution, which is given as [1]:

$I(z)=J_{o}\left[j \pi B \sqrt{1-\left(\frac{2 \mathrm{z}}{\mathrm{l}}\right)^{2}}\right] \quad-\frac{\mathrm{l}}{2} \leq \mathrm{z} \leq \frac{\mathrm{l}}{2}$

Where $J_{o}$ the Bessel function of the first kind of order zero is, $l$ is the total length of line source, and $B$ is the constant. The $B$ is also called one parameter. It was calculated by Hanson [1] as shown in table (1).

Table 1. The values of the constant B

\begin{tabular}{ccccccc}
\hline SLL(max) & $\mathbf{- 1 0}$ & $\mathbf{- 1 5}$ & $\mathbf{- 2 0}$ & $\mathbf{- 2 5}$ & $\mathbf{- 3 0}$ & $\mathbf{- 3 5}$ \\
\hline \multirow{2}{*}{ B } & $\begin{array}{c}\mathrm{j} 0.45 \\
97\end{array}$ & 0.3558 & 0.7386 & 1.0229 & $\begin{array}{c}1.27 \\
62\end{array}$ & 1.5136 \\
& & & & & & \\
\hline
\end{tabular}

For a liner array, the amplitude current excitation coefficient for $P$ odd or even number of elements can be calculated as:

$$
I_{n}=J_{o}\left[j \pi B \sqrt{1-\left(\frac{2 z_{n}}{p}\right)^{2}}\right]-\frac{p}{2} \leq z_{n} \leq \frac{p}{2}
$$

For $p=2 N+1($ odd $), z_{n}= \pm n d, n=1,2,3,4, \ldots \ldots, \frac{p}{2}$ when

$$
n=0,2 a_{1}=I_{1}\left(z_{0}\right) \rightarrow a_{1}=\frac{I_{1}(0)}{2}, z=0
$$

For

$$
\begin{aligned}
& p=2 N(\text { even }) \\
& z_{n}= \pm \frac{(2 n-1)}{2} d, n=1,2,3,4, \ldots \ldots, \frac{p}{2}
\end{aligned}
$$

Then the $z_{n}$ is the location of the $n$th elements on either side of the array $(\mathrm{d}$ is the separation space between elements in array ). The geometry and coordinates for an equally spaced array with odd elements is shown in figure (1), it consists of linear Long Backfire Antenna array equally spaced. 


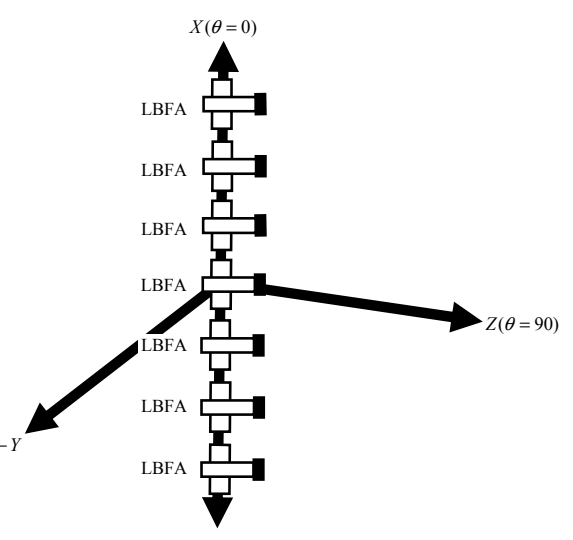

Figure 1. Linear LBFA array

\section{Linear Array Taylor Excitation}

For non uniform amplitude distribution and separation distance $(d=0.7 \lambda)$, the excitation coefficients for (11elements) of Binomial, Taylor amplitude distribution can be calculated as:

At SLLmax $=-30(\mathrm{~dB})=31.62, \quad \mathrm{~B}=1.2761$, number $\quad$ of elements $=11, l=7 \lambda$ :

$$
\begin{aligned}
& 2 a_{1}=I(z)_{z=0}=J_{0}(j \pi 4.0090)=11.3902 \rightarrow a_{1}=5.6951 . \\
& a_{2}=I(z)_{z= \pm 0.7 \lambda}=J_{0}(j \pi 3.928)=10.6216 \\
& a_{3}=I(z)_{z= \pm 1.4 \lambda}=J_{0}(j \pi 3.6743)=8.5497 \\
& a_{4}=I(z)_{z= \pm 2.1 \lambda}=J_{0}(j \pi 3.2072)=5.7814 \\
& a_{5}=I(z)_{z= \pm 2.8 \lambda}=J_{0}(j \pi 2.4054)=3.0617 \\
& a_{6}=I(z)_{z= \pm 3.5 \lambda}=J_{0}(j \pi 0)=1 .
\end{aligned}
$$

\begin{tabular}{lcccccc}
\hline $\begin{array}{l}\text { Excitation } \\
\begin{array}{l}\text { Coefficient } \\
\text { Taylor }\end{array}\end{array}$ & $a_{1}$ & $a_{2}$ & $a_{3}$ & $a_{4}$ & $a_{5}$ & $a_{6}$ \\
distribution & 5.6951 & 10.6216 & 8.5497 & 5.7814 & 3.0617 & 1 \\
\hline
\end{tabular}

Taylor array factor for (11 elements) and space separated ( $0.7 \lambda$ ) can be represented by the equations (3)

$$
\begin{array}{rl}
A F_{11}=5.6951+10 & 6216 \cos (1.4 \pi \cos (\theta)) \\
& +8.5497 \cos (2.8 \pi \cos (\theta)) \\
& +5.7814 \cos (4.2 \pi \cos (\theta)) \\
& +3.0617 \cos (5.6 \pi \cos (\theta)) \\
& +\cos (7 \pi \cos (\theta))
\end{array}
$$

Since the array is broad side $(d<\lambda)$ the array factor pattern must has maximum radiation pattern at $\theta=90$ ( toward Z-direction) normal to the plan of array location (X-Y plan ) as shown in figure (2).

It is clear that side lobes level for Taylor array are tapering sides lobe(not equal level as in Chebyshev array)[7], on other meaning the directivity in Taylor arrays haven't limitation rang by increasing the length of the arrays ( increasing number of elements in the array).

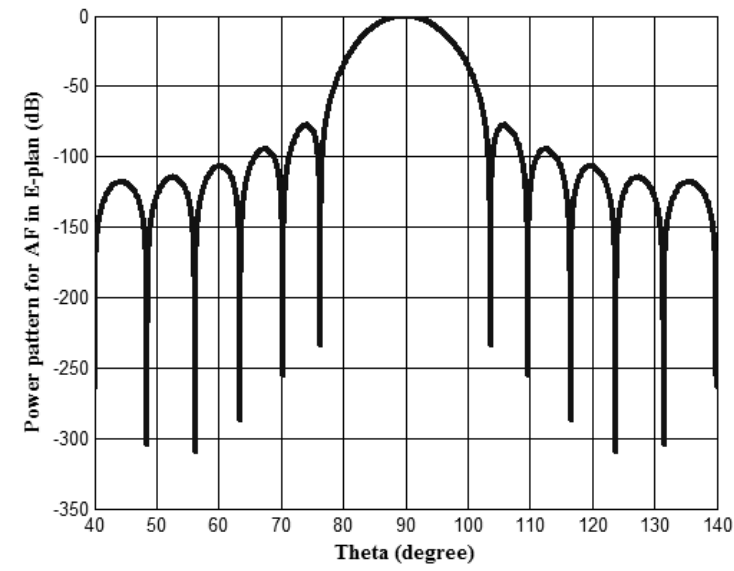

Figure 2. power pattern for Array factor Taylor distribution (11)elements

Multiplying the Taylor array factor in equation (3) by the pattern for single element Long backfire antenna in E-plan $[2,3,4,5]$, the total radiation pattern in for (11) Long backfire antennas Taylor distribution with space separation $(d=0.7 \lambda)$ can be represented in figure (3). Since the LBFAs (long Backfire antennas) are located along (Xaxis )the radiation pattern in E-plan $(\theta=90)$ will has narrow beam width compared with radiation pattern in $\mathrm{H}$ plan $(\theta=0)[1,2]$.The total radiation pattern for Taylor array in figure $(4)$ has maximum directivity $(25.5 \mathrm{~dB})$ and SLL max $(-86 \mathrm{~dB})$.

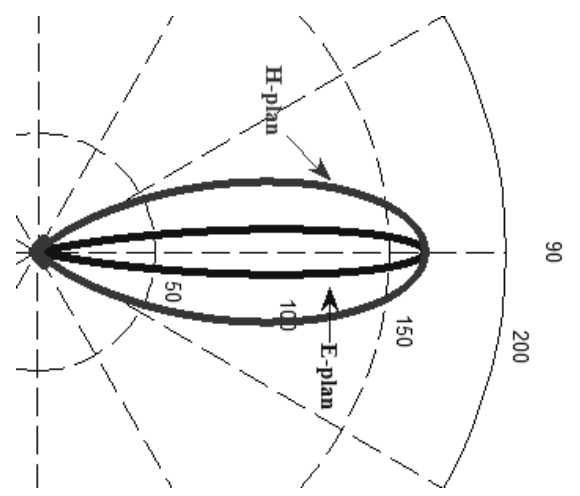

Figure 3. Total radiation pattern for Taylor array(11)LBFAs

By changing the space separation between elements in linear array Taylor excitation (11 elements) the directivity will be changed according to the space dimension (d) because the length of the array $(l=(n-1) d)$ and number of side lobe will be changed also[ 7] as shown in figure (4), when the separation distance $(d)$ becomes less than $(0.3 \lambda)$ the radiation pattern will take the shape of radiation pattern for single LBFA due to mutual coupling [7]. 


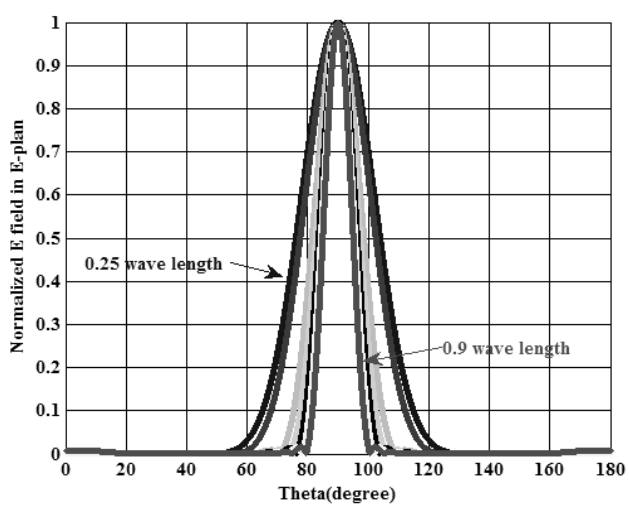

Figure 4. Taylor array (11)elements with different spaces $(0.25 \lambda-0.9 \lambda)$

From figure (4) it is clear that by increasing the separation distance to $(\mathrm{d}=0.9 \lambda)$ new sides lobe will create at ( $\theta=0$ and $\theta=180)$ because the array becomes almost end fire array [6 ].All the calculation results for total radiation pattern are illustrated in table (2).

Table 2. Directivity and SLL max for Taylor array $(d=0.1 \lambda-0.9 \lambda)$

\begin{tabular}{lllll}
\hline space & $\begin{array}{c}\boldsymbol{\theta}_{\mathbf{E}} \\
(\text { degree })\end{array}$ & $\begin{array}{c}\boldsymbol{\theta}_{\mathbf{H}} \\
(\text { degree })\end{array}$ & $\begin{array}{l}\text { SLL max } \\
(\mathbf{d B})\end{array}$ & D (dB) \\
\hline $0.9 \lambda$ & 4.8 & 18.8 & -82.7 & 26.6 \\
$0.8 \lambda$ & 5.35 & 18.8 & -84 & 26.13 \\
$0.7 \lambda$ & 6.1 & 18.8 & -86 & 25.5 \\
$0.6 \lambda$ & 6.95 & 18.8 & -89 & 25 \\
$0.5 \lambda$ & 8.1 & 18.8 & -95 & 24.32 \\
$0.3 \lambda$ & 11.72 & 18.8 & -133 & 22.72 \\
$0.25 \lambda$ & 12.8 & 18.8 & -188.66 & 22.34 \\
$0.1 \lambda$ & 17.38 & 18.8 & -80 & 21 \\
\hline
\end{tabular}

As the number of elements in Taylor array reduced the total length of array will decreased which caused decreasing the directivity and number of side lobes as shown in figure (5). Using numerical methods, new formula for theoretical directivity calculation can be obtained as a function of space separation (d) and number of elements in the array :

$$
D_{\text {array factor }}=1.8 \frac{(N-1) d}{\lambda}
$$

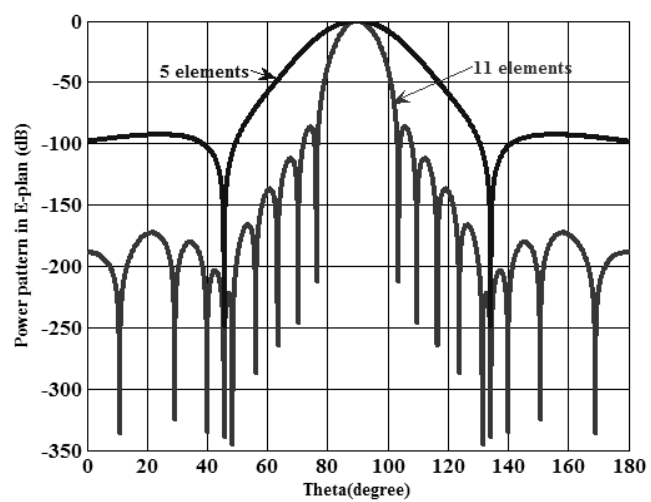

Figure 5. Power pattern for Taylor arrays LBFA (11 and 5 elements).

\section{Comparison between Different Types of Non Uniform Excitation}

For same number of elements (11) and desired side lobe level $(-30 \mathrm{~dB})$ with different space separation $(\mathrm{d})$ the radiation characteristics especially (directivity and SLL max) for linear array LBFA Taylor excitation will be compared with another types of excitation (Chebyshev and binomial ) [7 ] as shown in table (3).

From table (3) it is clear that the directivity is almost the same value for all types of non uniform excitation (Taylor ,Chbeyshev and Binomial) for separation distance $(d<0.3 \lambda)$ because the field lengths (A2e) for each elements will over lab with the(A2e) for the neighbor LBFA which caused the arrays became similar to one large LBFA [7,],also SLL max for the three types of excitation are almost closed for $(d=0.25 \lambda$ and $0.1 \lambda)$ except SLL max for Taylor array $(-188.66 \mathrm{~dB})$ for $(\mathrm{d}=0.25 \lambda)$.

Table 3. Directivity and SLL max for (Taylor, Chebyshev and Binomial) LBFA arrays

\begin{tabular}{|c|c|c|c|c|}
\hline space & properties & Taylor & Chebyshev & Binomial \\
\hline \multirow[b]{2}{*}{$0.9 \lambda$} & $\mathrm{D}(\mathrm{dB})$ & 26.6 & 27 & 23.6 \\
\hline & $\begin{array}{l}\text { SLL } \\
\max (d B)\end{array}$ & -82.7 & -72.67 & -85 \\
\hline \multirow[b]{2}{*}{$0.8 \lambda$} & $\mathrm{D}(\mathrm{dB})$ & 26.13 & 26.6 & 23 \\
\hline & $\begin{array}{l}\text { SLL } \\
\max (d B)\end{array}$ & -84 & -73.6 & -117.5 \\
\hline \multirow[b]{2}{*}{$0.7 \lambda$} & $\mathrm{D}(\mathrm{dB})$ & 25.5 & 26 & 22.5 \\
\hline & $\begin{array}{l}\text { SLL } \\
\max (d B)\end{array}$ & -89 & -75 & -836.2 \\
\hline \multirow[b]{2}{*}{$0.5 \lambda$} & $\mathrm{D}(\mathrm{dB})$ & 24.32 & 24.7 & 21.388 \\
\hline & $\begin{array}{l}\text { SLL } \\
\max (d B)\end{array}$ & -95 & -80.9 & -261.5 \\
\hline \multirow[b]{2}{*}{$0.25 \lambda$} & $\mathrm{D}(\mathrm{dB})$ & 22.34 & 22.5 & 19.795 \\
\hline & $\begin{array}{l}\text { SLL } \\
\max (d B)\end{array}$ & -188.66 & -125 & -110 \\
\hline \multirow[b]{2}{*}{$0.1 \lambda$} & $\mathrm{D}(\mathrm{dB})$ & 21 & 21.83 & 19 \\
\hline & $\begin{array}{l}\text { SLL } \\
\max (d B)\end{array}$ & -80 & -81 & -72.2 \\
\hline
\end{tabular}

Directivity for Taylor LBFA arrays is better than directivity for Binomial LBFA arrays and closed to value of directivity for Chebyshev LBFA arrays for space $(\mathrm{d}=\mathbf{0 . 5} \lambda$ to $\mathrm{d}=\mathbf{0 . 9} \lambda$ ) while (SLL max) for Chebyshev arrays are better than (SLL max) for Taylor arrays .

SLL max for Chebyshev arrays are almost constant while SLL max for Taylor and Binomial arrays are varied according to change the length of these arrays .The optimized space separation for Chebyshev array is $(\mathrm{d}=\mathbf{0 . 8} \boldsymbol{\lambda})$ while the optimized space for Taylor array is $(\mathrm{d}=\mathbf{0 . 5} \boldsymbol{\lambda})$ with $(\mathrm{SLL} \max =-95 \mathrm{~dB}$ and directivity $=24.32 \mathrm{~dB})$ compared with $(\mathrm{d}=\mathbf{0 . 7} \boldsymbol{\lambda})$ as optimum space for Binomial array. For long Chebyshev arrays ( $\mathrm{Nd}>100 \lambda)$ the directivity has limitation rang (as shown in fig. 4) because increasing number of elements in Chebyshev array will create new lobes with same level but in Taylor arrays the new created 
lobes in long arrays are tapering side lobes so the directivity will not had limitation rang .

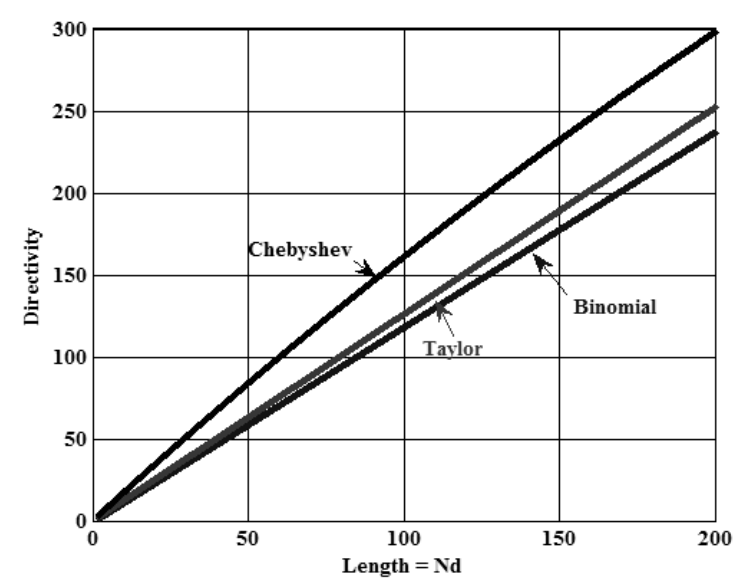

Figure 6. Directivity for (AF)against arrays length (Chebyshev, Taylor and Binomial) with (SLL $\max =-30 \mathrm{~dB}$ )

\section{Conclusion}

The center excitation coefficient $\left(a_{1}=5.6951\right)$ for all odd Taylor arrays which had same desired side lobe level $(-30 \mathrm{~dB})$ whatever the length of the array ,and for even Taylor arrays the center coefficient must be equal to $\left(a_{1}=11.3902\right)$.

The edge excitation coefficient $\left(a_{N}=1\right)$ for all Taylor arrays odd and even whatever the length of the array. Asymmetrical amplitude distribution between center and edge of Taylor arrays produce lower side lobe and heigh directivity.

The directivity in Taylor arrays had no limitation rang by increasing the length of the array due to the new tapering side lobes which are created when number of elements in the array was increased on contrary what happened in Chebyshev arrays .

Directivity in Taylor arrays is much better than the directivity in Binomial arrays and almost similar to the directivity in Chebyshev arrays but the last type of excitation (Chebyshev) had lower side lobe level.

\section{References}

[1] Robert S. Elliot "Antenna theory and design, John Wiley and sons: Hoboken, New Je2003.

[2] Amer Tawfeeq Abed " Backfire Antenna Theory and Design", LAP LAMBERT for Academic PublicationGermany 2013

[3] Amer Tawfeeq Abed, Developing and designing LBF Antenna as a single element and linear array uniform amplitude and space in $(X$-band), Canadian Journal on Electrical and Electronics Engineering Vol. 2, No. 2, August 2011

[4] Amer Tawfeeq Abed, Chandan Kumar, Design and optimization Long Backfire Antenna in(C- band) using Hrestove equations, Canadian Journal on Electrical and Electronics Engineering Vol. 2, No. 10, October 2011.

[5] Amer Tawfeeq Abed "Effect of changing length on radiation properties for backfire antennas" Canadian Journal on Electrical and Electronics Engineering Vol. 2, No. 10, October 2011.

[6] Amer Tawfeeq Abed, Directivity In Linear Arrays Uniform Space and Distribution, Canadian Journal on Electrical and Electronics Engineering Vol. 3, No. 7, September 2012.

[7] Amer Tawfeeq Abed, Improving Directivity and SLL max in Uniform Space and Non Uniform Excitation Antenna Arrays , Canadian Journal on Electrical and Electronics Engineering Vol. 3, No. 8, October 2012.

\section{Biography}

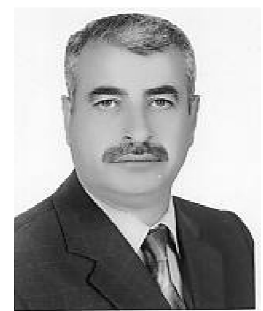

Amer Tawfeeq Abed: Bach in electrical eng. university of Baghdad 1984 Has wide experience in designing RF circuit and Antenna field ...Master communication Eng. University Tenaga(UNITEN) Malaysia in 2012. Now lecturer in ALMAMON University Collegecommunication eng. dept 